ADP-00-38/T421

hep-lat/0008018

\title{
Incorporating Chiral Symmetry and Heavy Quark Theory in Extrapolations of Octet Baryon Charge Radii
}

\author{
E.J. Hackett-Jones* , D.B. Leinweber ${ }^{\dagger}$ and A.W. Thomas ${ }^{\ddagger}$ \\ Special Research Centre for the Subatomic Structure of Matter and \\ Department of Physics and Mathematical Physics, University of Adelaide, Australia 5005
}

\begin{abstract}
We extrapolate lattice calculations of electric charge radii of the spin- $1 / 2$ baryon octet to the physical regime. The extrapolation procedure incorporates chiral perturbation theory and heavy quark effective theory in the appropriate limits. In particular, this procedure includes the non-analytic, logarithmic terms from pion loops. The electric charge radii of the nucleons and $\Sigma^{-}$obtained from the chiral extrapolations agree well with experimental data. We make predictions for the charge radii of the remaining baryons in anticipation of future experimental measurements.
\end{abstract}

*ehackett@physics.adelaide.edu.au

$\dagger$ †leinweb@physics.adelaide.edu.au

‡athomas@physics.adelaide.edu.au 


\section{INTRODUCTION}

Lattice QCD is so far the most successful method of calculating hadronic observables from the theory of QCD. However, computational limitations mean that hadronic observables are calculated on the lattice at quark masses larger than their physical values. Hence results from lattice simulations cannot be directly compared with experimental data. Although, with improvements in actions, algorithms and computer speed, future lattice calculations will be performed much closer to the physical regime, these improvements will proceed over many years. Therefore, to make sense of any lattice results produced to date, and to compare them with experiment, one must understand how to extrapolate lattice results, obtained at large quark masses, to the physical world.

One of the difficulties with calculating hadronic observables at heavy quark masses on the lattice is that chiral perturbation theory cannot be applied in this quark mass regime. However, chiral expansions of hadronic observables contain important non-analytic terms as a function of the quark mass, $m_{q}$ (or equivalently of $m_{\pi}^{2}$, as $m_{q} \propto m_{\pi}^{2}$ in this range). It is vital that this non-analytic behaviour is included in any extrapolation to the physical regime [1]- [8].

The chiral expansion of the squared electric charge radius of a spin- $1 / 2$ octet baryon includes non-analytic behaviour in the form of logarithmic terms in $m_{\pi}$ (or $m_{q}$ ). To extrapolate the lattice results for electric charge radii we incorporate these logarithmic terms in our extrapolation formulae, while ensuring that the correct heavy quark behaviour is also maintained. A similar approach [1] has been successful in explaining why lattice simulations of pion and proton charge radii are similar in size, while experimental measurements reveal a significant difference. The dramatic differences in the chiral behaviour of the pion and proton charge radii account for the similarity of the lattice results at moderately heavy pion masses, while allowing good agreement with experiment at the physical pion mass. This illustrates the importance of including meson cloud effects in extrapolations of lattice results to the physical regime. In this paper, we improve the formalism of chiral extrapolations by incorporating both chiral symmetry and heavy quark effective theory.

Our lattice "data" for the electric charge radii is taken from the calculations of Ref. [9]. The contributions to the charge radii from individual quark flavours are also given there. These results are the only available lattice calculations of the electric charge radii of spin- $1 / 2$ octet baryons. For each baryon, the electric charge radius was calculated at three different quark masses, corresponding to rather heavy pion masses (all exceeding $600 \mathrm{MeV}$ ). Here we extrapolate these results as functions of the squared pion mass, $m_{\pi}^{2}$, to obtain predictions for the charge radii at the physical pion mass $(139 \mathrm{MeV})$. Because the lattice calculations are quenched, we expect that there are errors in the lattice data which we have been unable to take into account. However, we expect that errors from the quenching approximation

will be rather small. At the quark masses considered on the lattice, the dominant effect is a simple renormalization of the strong coupling constant, accounted for in setting the lattice spacing scale. When results from unquenched simulations become available, the formalism presented here may be readily applied. 


\section{EXTRAPOLATIONS}

Dynamical breaking of chiral symmetry in the QCD lagrangian results in the formation of an octet of (pseudo-) Goldstone bosons. Goldstone boson loops give rise to significant non-analytic behaviour in hadronic observables, such as $\left\langle r_{i}{ }^{2}\right\rangle$ and magnetic moments, as a function of the quark mass, $m_{q}$. Using an expansion about the chiral SU(3) limit gives the following expression for the squared electric charge radius, $\left\langle r_{i}{ }^{2}\right\rangle$, of a spin-1/2 octet baryon (labelled by $i$ ) 10

$$
\left\langle r_{i}^{2}\right\rangle=\gamma_{i}+\sum_{X=\pi, K} \frac{6 \alpha_{i}^{(X)}}{\left(4 \pi f_{\pi}\right)^{2}} \ln \left(\frac{m_{X}}{\lambda}\right)+\ldots
$$

Here $f_{\pi}$ is the pion decay constant $(93 \mathrm{MeV})$ and $\lambda$ is the scale of the dimensional regularization. (The value of $\gamma_{i}$ is clearly correlated with the choice of $\lambda$.) Unlike $\mathrm{SU}(2)$-flavour symmetry, $\mathrm{SU}(3)$ is significantly broken in the physical world, with the strange quark mass the same order of magnitude as $\Lambda_{\mathrm{QCD}}$ for low-energy phenomenology. The squared kaon mass exceeds the squared pion mass by over an order of magnitude. Given that the source of the meson cloud associated with a baryon is of a finite size, one might anticipate that the role of the kaon cloud will be suppressed away from the $\mathrm{SU}(3)$ chiral limit [11]. The form factor describing the finite size of the kaon source will act to suppress kaon loop effects like $m_{X}^{-4}$ at large $m_{X}$ (comparable to $m_{K}$ ). This was demonstrated within a particular chiral quark model for the nucleon magnetic moments in Ref. [4]. Despite the model-dependence associated with the form factors, the lattice results themselves are very slowly varying functions of $m_{X}$ at values of the order $m_{K}$ or higher, thus supporting the general conclusion. Therefore kaon loop effects are expected to be small and slowly varying as a function of $m_{q}$. Hence we do not explicitly include kaon contributions in our extrapolation formulae. Conversely, since $m_{\pi}$ varies rapidly with $m_{q}$, the leading non-analytic behaviour in $m_{\pi}$ must be included explicitly in an extrapolation to the chiral regime.

To extrapolate the lattice calculations of the electric charge radii of the spin- $1 / 2$ octet baryons, we consider two distinct fitting procedures. Both these extrapolation schemes satisfy the constraints of chiral perturbation theory and heavy quark effective theory. The first extrapolation procedure we investigate is given simply by the formula

$$
\left\langle{r_{i}}^{2}\right\rangle=\frac{c_{1}+\chi_{i} \ln \left(m_{\pi} / \Lambda\right)}{1+c_{2} m_{\pi}^{2}}
$$

where $\left\langle r_{i}{ }^{2}\right\rangle$ are the lattice QCD results (at several values of $m_{\pi}$ ) extracted from Ref. [9], $c_{1}$ and $c_{2}$ are fit parameters chosen to best fit these results, $\chi_{i}$ (corresponding to the $i^{\text {th }}$ baryon) is fixed (model-independently) by chiral perturbation theory and $\Lambda$, which is directly correlated with $c_{1}$, is fixed at $1 \mathrm{GeV}$. This extrapolation procedure is not feasible for the neutral baryons because $\left\langle r_{i}{ }^{2}\right\rangle \rightarrow 0$ as $m_{\pi}$ becomes large and thus sensitivity to the $c_{2}$ fit parameter is lost. In order to extrapolate the neutral baryon charge radii results we consider a second extrapolation procedure, focusing on individual quark "sector" (or quark flavour) contributions, as discussed below. 
Clearly the extrapolation formula given in Eq. (2) builds in the correct chiral behaviour in the $\mathrm{SU}(2)$ limit, since in the limit $m_{\pi} \rightarrow 0$ it can be expanded as follows

$$
\left\langle r_{i}^{2}\right\rangle=c_{1}+\chi_{i} \ln \left(m_{\pi}\right)-c_{2} m_{\pi}^{2}+\ldots
$$

(Recall that the scale $\Lambda$, in Eq. (2), has been set to $1 \mathrm{GeV}$. This choice is also implicit in Eqs. (6)-(10), below, where $m_{\pi}$ in the logarithm must be in GeV.) This agrees with the

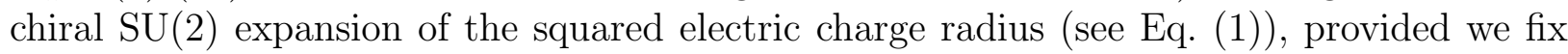
the coefficient $\chi_{i}$ to $6 \alpha_{i}^{(\pi)} /\left(4 \pi f_{\pi}\right)^{2}$ for the $i^{\text {th }}$ baryon. The coefficients $\alpha_{i}^{(\pi)}$ and $\chi_{i}$ are given in Table [.

In the large $m_{q}$ limit, we expect that the quarks behave non-relativistically, and the squared charge radius falls off as $m_{q}^{-2}$, as it does in non-relativistic quantum mechanics. In the region where $m_{q}$ is very large, $m_{q} \propto m_{\pi}$, and hence we require that

$$
\left\langle r_{i}^{2}\right\rangle \propto \frac{1}{m_{\pi}^{2}}
$$

as $m_{\pi}$ becomes extremely large. This is clearly satisfied by our first extrapolation formula, Eq. (2), since the logarithm is very slowly varying.

In the second extrapolation procedure we deal separately with the individual quark sector contributions to the baryon charge radii. For example, in the case of the nucleons, we extrapolate the up and down sector contributions separately. For the hyperons the strange and light sector results are extrapolated separately. This avoids the problem encountered with the neutral baryons which was mentioned previously, because now all the quantities being extrapolated are charged, even if the overall charge on the baryon is zero. This separation is valid because the squared electric charge radius can be decomposed as

$$
\left\langle r_{i}^{2}\right\rangle=\sum_{q=u, d, s} e_{q}\left\langle r_{i}^{(q) 2}\right\rangle
$$

where $\left\langle r_{i}^{(q) 2}\right\rangle$ is the contribution from the $q^{\text {th }}$ quark sector and $e_{q}$ is the charge of this quark sector. Therefore, provided that the extrapolation formulae from each sector add so that the chiral and heavy quark limits of the sum are in agreement with Eqs. (11) and (田) respectively, this method contains the same physics as the first method, but simply makes use of the extra information contained in the individual quark sector results. Not only does this second extrapolation procedure solve the neutral baryon extrapolation difficulty, it also provides predictions for individual quark sector radii, which will be resolved at Jefferson Lab for the nucleon [12], and perhaps future accelerator facilities for hyperons.

Isolation of the individual quark sector contributions to the charge radii is relatively straightforward from the theoretical point of view. For example, to isolate the $u$-sector contribution to the charge radius of the proton one simply sets the $d$-quark charge to zero and calculates the proton charge radius as if only the $u$ quark carried charge. In the chiral expansion of the proton charge radius, the coefficient of the logarithm, $\chi_{p}^{(\pi)}$, originates from the pion loop, $p \rightarrow n+\pi^{+}$(see Fig. [1), and includes the charge of the pion cloud. Therefore, to 


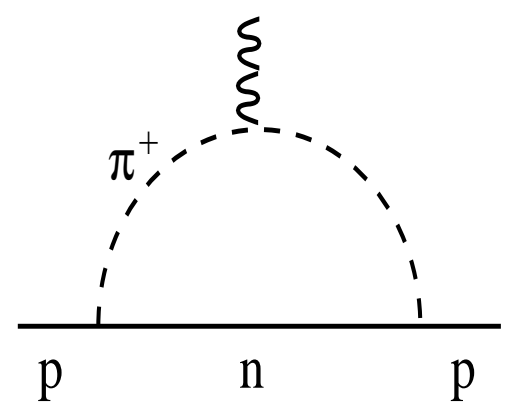

FIG. 1. Schematic illustration of the pion loop which produces the leading non-analytic contribution to the proton charge radius.

extrapolate the $u$-sector contribution to the proton charge radius, the appropriate coefficient of the logarithm is $\frac{2}{3} \chi_{p}^{(\pi)}$, since the pion now carries charge $+2 / 3$. Thus we extrapolate the $u$-sector results of the proton according to

$$
2 e_{u}\left\langle r_{p}^{(u) 2}\right\rangle=\frac{c_{1}+\frac{2}{3} \chi_{p}^{(\pi)} \ln \left(m_{\pi}\right)}{1+c_{2} m_{\pi}^{2}}
$$

where $\chi_{p}^{(\pi)}$ is the full chiral coefficient of the proton, given in Table $\llbracket$ and $\left\langle r_{p}^{(u) 2}\right\rangle$ is the squared charge radius of a single $u$-quark of unit charge. Similarly, the $d$-sector results are extrapolated according to

$$
e_{d}\left\langle r_{p}^{(d) 2}\right\rangle=\frac{c_{1}{ }^{\prime}+\frac{1}{3} \chi_{p} \ln \left(m_{\pi}\right)}{1+c_{2}{ }^{\prime} m_{\pi}^{2}},
$$

where the factor of $1 / 3$ originates from the $\bar{d}$ contribution to the pion cloud. Clearly adding the left hand sides of Eqs. (6) and (7) yields the full expression for $\left\langle r_{p}^{2}\right\rangle$. In the chiral limit the right hand sides add so that the correct chiral form for $\left\langle r_{p}^{2}\right\rangle$, given in Eq. (3), is retained. The sum of Eqs. (6) and (7) also obeys the correct heavy quark behaviour, given in Eq. (田). Since the parameters $c_{2}$ and $c_{2}{ }^{\prime}$ are not necessarily the same, the individual quark sector extrapolation formulae (Eqs. (6) and (7)) cannot be added directly to give Eq. (2). Therefore, in general we do not expect the two extrapolation procedures to give exactly the same results. For the charged baryons this may be used to help quantify the systematic error of the approach. For the neutron, the analogous extrapolation functions are given by

$$
e_{u}\left\langle r_{n}^{(u) 2}\right\rangle=\frac{c_{1}+\frac{2}{3} \chi_{n}^{(\pi)} \ln \left(m_{\pi}\right)}{1+c_{2} m_{\pi}^{2}},
$$

for the $u$-quark sector, and

$$
2 e_{d}\left\langle r_{n}^{(d) 2}\right\rangle=\frac{c_{1}{ }^{\prime}+\frac{1}{3} \chi_{n}^{(\pi)} \ln \left(m_{\pi}\right)}{1+c_{2}{ }^{\prime} m_{\pi}^{2}},
$$

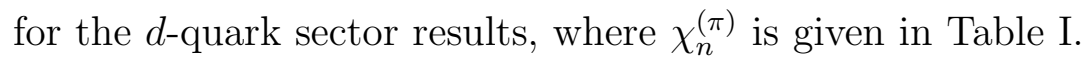


We now consider extrapolating the hyperon charge radii results using the second extrapolation procedure, i.e., extrapolating the strange and light quark sector results separately. In extrapolating the strange quark sector the charges of the light quarks are set to zero. Since the logarithmic term considered here originates from pionic corrections to the charge radius (and pions do not contain strange quarks), the coefficient of the logarithm in the strange sector extrapolation will be zero. Similarly, for the light sector extrapolation, strange quarks do not carry charge and hence the coefficient of the logarithm in this extrapolation will be the full coefficient, $\chi_{i}^{(\pi)}$. This results in the following extrapolation formulae for the hyperon quark sector contributions

$$
e_{l}\left\langle r_{i}^{(l) 2}\right\rangle=\frac{c_{1}+\chi_{i} \ln \left(m_{\pi}\right)}{1+c_{2} m_{\pi}^{2}}
$$

and

$$
e_{s}\left\langle r_{i}^{(s) 2}\right\rangle=\frac{c_{1}^{\prime}}{1+c_{2}^{\prime} m_{\pi}^{2}}
$$

where $i$ runs over the hyperons only, and $l$ corresponds to the light-quark ( $u$ and/or $d$ ) sector. Since the strange quark mass is held fixed in the light quark mass extrapolation, any variation in the strange quark sector is purely an environment effect from the surrounding light quarks. As such, the functional form for the strange quark sector is constrained by neither leading order chiral perturbation theory nor heavy quark effective theory. As we shall see, $c_{2}{ }^{\prime}$ is small and negative for each hyperon, which suggests that a simple linear ansatz for the strange quark sector extrapolation could also have been used.

\section{RESULTS}

The lattice QCD simulations were performed on a $24 \times 12 \times 12 \times 24$ periodic lattice using standard Wilson actions at $\beta=5.9$. Dirichlet boundary conditions were used for fermions in the time direction. Twenty-eight quenched gauge configurations were generated by the Cabibbo-Marinari 18 pseudo-heat-bath method. The conserved vector current was derived from the Wilson fermion action via the Noether procedure. The associated lattice Ward identity protects this vector current from renormalization. The radii were produced by fitting the electric form factor to dipole and monopole forms, allowing a charge radius to be extracted in each case. Since it is known from experiment that the dipole form is more suitable for parameterizing the electric form factor, we consider only the dipole results here. Statistical uncertainties in the lattice simulation results are calculated in a third-order, single elimination jackknife [19,20]. Further details may be found in Ref. [9].

The extrapolations of lattice calculations for the charge radii of the spin- $1 / 2$ baryon octet are shown in Figs. 267. Extrapolations of baryon charge radii results, performed according to Eq. (2), are indicated by the solid lines, where the full circles (•) represent the baryon charge radii from lattice QCD and the extrapolated value at $m_{\pi}=139 \mathrm{MeV}$. The individual quark sector extrapolations are shown by the dashed and dot-dashed lines, and the baryon 


\begin{tabular}{|c|c|c|c|c|c|c|}
\hline Baryon or Quark Sector & $\alpha_{i}^{(\pi)}$ & $\chi_{i}$ & $c_{1}$ & $c_{2}$ & $\left\langle r^{2}\right\rangle$ & Experiment \\
\hline 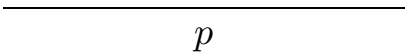 & $-\frac{1}{6}-\frac{5}{6}(D+F)^{2}$ & -0.174 & 0.34 & 0.50 & $0.68(8)$ & $0.740(15)$ 114 \\
\hline$u_{p}$ & $\frac{2}{3}\left[-\frac{1}{6}-\frac{5}{6}(D+F)^{2}\right]$ & -0.116 & 0.52 & 0.73 & $0.74(11)$ & \\
\hline$d_{p}$ & $\frac{1}{3}\left[-\frac{1}{6}-\frac{5}{6}(D+F)^{2}\right]$ & -0.058 & -0.18 & 1.38 & $-0.06(5)$ & \\
\hline${ }^{\star} p$ & & & & & $0.68(10)$ & $0.740(15)$ 14 \\
\hline$n$ & $\frac{1}{6}+\frac{5}{6}(D+F)^{2}$ & 0.174 & & & & \\
\hline$u_{n}$ & $\frac{2}{3}\left[\frac{1}{6}+\frac{5}{6}(D+F)^{2}\right]$ & 0.116 & 0.35 & 1.38 & $0.12(10)$ & \\
\hline$d_{n}$ & $\frac{1}{3}\left[\frac{1}{6}+\frac{5}{6}(D+F)^{2}\right]$ & 0.058 & -0.26 & 0.73 & $-0.37(6)$ & \\
\hline${ }^{\star} n$ & & & & & $-0.25(8)$ & $-0.113(4)$ 15 \\
\hline$\Lambda$ & 0 & 0 & & & & \\
\hline$l_{\Lambda}$ & 0 & 0 & 0.15 & 0.97 & $0.14(3)$ & \\
\hline$s_{\Lambda}$ & 0 & 0 & -0.07 & -0.10 & $-0.07(1)$ & \\
\hline${ }^{\star} \Lambda$ & & & & & $0.07(3)$ & \\
\hline$\Sigma^{+}$ & $-\frac{1}{3}-\frac{5}{3}\left(\frac{D^{2}}{3}+F^{2}\right)$ & -0.138 & 0.68 & 2.03 & $0.92(11)$ & \\
\hline$l_{\Sigma^{+}}$ & $-\frac{1}{3}-\frac{5}{3}\left(\frac{D^{2}}{3}+F^{2}\right)$ & -0.138 & 0.58 & 0.93 & $0.83(8)$ & \\
\hline$s_{\Sigma^{+}}$ & 0 & 0 & -0.06 & -0.17 & $-0.06(1)$ & \\
\hline${ }^{\star} \Sigma^{+}$ & & & & & $0.77(8)$ & \\
\hline$\Sigma^{0}$ & 0 & 0 & & & & \\
\hline$l_{\Sigma^{0}}$ & 0 & 0 & 0.19 & 1.48 & $0.18(2)$ & \\
\hline$s_{\Sigma^{0}}$ & 0 & 0 & -0.06 & -0.17 & $-0.06(1)$ & \\
\hline${ }^{\star} \Sigma^{0}$ & & & & & $0.12(2)$ & \\
\hline$\overline{\Sigma^{-}}$ & $\frac{1}{3}+\frac{5}{3}\left(\frac{D^{2}}{3}+F^{2}\right)$ & 0.138 & -0.25 & 0.08 & $-0.52(3)$ & $-0.60(16) 16$ \\
\hline & & & & & & $-0.91(72) 17$ \\
\hline$l_{\Sigma^{-}}$ & $\frac{1}{3}+\frac{5}{3}\left(\frac{D^{2}}{3}+F^{2}\right)$ & 0.138 & -0.21 & 0.37 & $-0.47(3)$ & \\
\hline$s_{\Sigma^{-}}$ & 0 & 0 & -0.06 & -0.17 & $-0.06(1)$ & \\
\hline${ }^{\star} \Sigma^{-}$ & & & & & $-0.54(3)$ & $-0.60(16) 16$ \\
\hline & & & & & & $-0.91(72) \sqrt{17}$ \\
\hline$\Xi^{0}$ & $-\frac{1}{6}-\frac{5}{6}(D-F)^{2}$ & -0.035 & & & & \\
\hline$l_{\Xi^{0}}$ & $-\frac{1}{6}-\frac{5}{6}(D-F)^{2}$ & -0.035 & 0.29 & 0.97 & $0.35(5)$ & \\
\hline$s_{\Xi^{0}}$ & 0 & 0 & -0.15 & -0.05 & $-0.15(1)$ & \\
\hline${ }^{\star} \Xi^{0}$ & & & & & $0.20(5)$ & \\
\hline$\Xi^{-}$ & $\frac{1}{6}+\frac{5}{6}(D-F)^{2}$ & 0.035 & -0.24 & 0.07 & $-0.32(2)$ & \\
\hline$l_{\Xi^{-}}$ & $\frac{1}{6}+\frac{5}{6}(D-F)^{2}$ & 0.035 & -0.12 & 0.70 & $-0.19(2)$ & \\
\hline$s_{\Xi^{-}}$ & 0 & 0 & -0.15 & -0.05 & $-0.15(1)$ & \\
\hline${ }^{\star} \Xi^{-}$ & & & & & $-0.34(2)$ & \\
\hline
\end{tabular}

TABLE I. Baryon electric charge radii and the quark sector contributions. The latter are defined on the left hand sides of Eqs. (6)-(11). One-loop corrected estimates of $\alpha_{i}^{(\pi)}$ (in Eq. (11)) and $\chi_{i}$ (in units of $\mathrm{fm}^{2}$ ) for each octet baryon are indicated. For each extrapolation, the fit parameters, $c_{1}$ and $c_{2}$, and the predicted value of $\left\langle r^{2}\right\rangle$ at the physical pion mass are reported. Asterisks denote the squared charge radii reconstructed from the sum of separate quark sector extrapolations. (The units are such that the pion mass is in $\mathrm{GeV}$ and the squared charge radius in $\mathrm{fm}^{2}$.) 


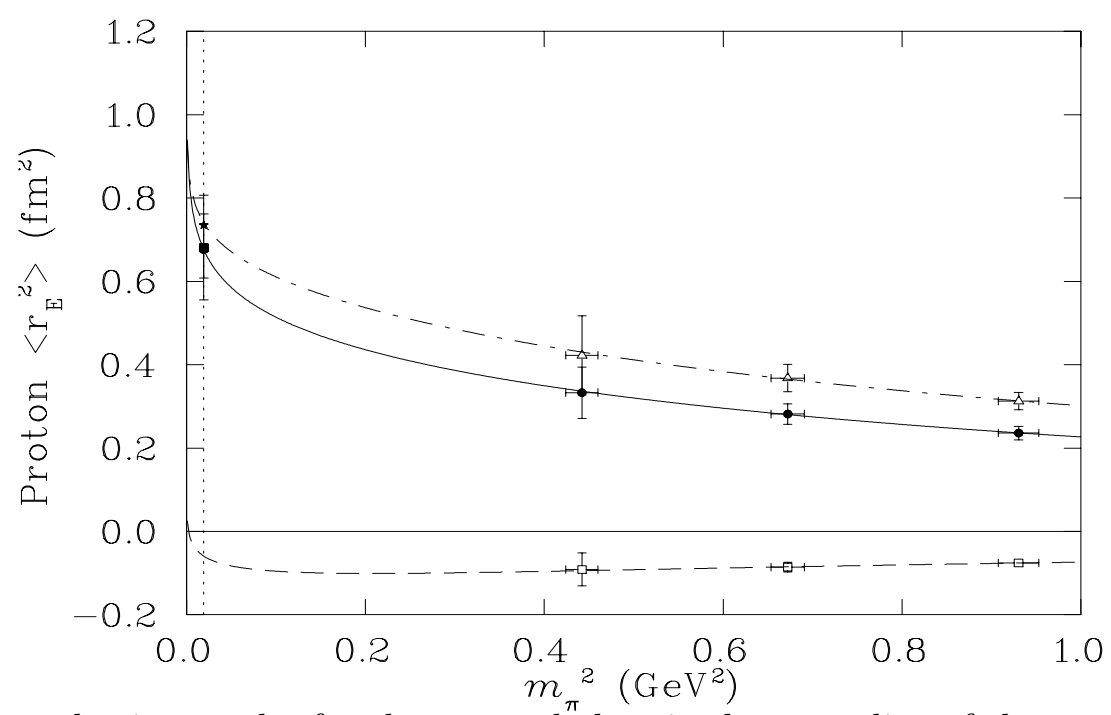

FIG. 2. Fits to lattice results for the squared ${ }^{\pi}$ electric charge radius of the proton. Fits to the individual quark sector results are also shown. The $u$-quark sector results are indicated by open triangles and the $d$-quark sector results by open squares. Physical values predicted by the fits are indicated at the physical pion mass, where the full circle denotes the result predicted from the first extrapolation procedure and the full square denotes the baryon radius reconstructed from the quark sector extrapolations (see text). (N.B. The latter values are actually so close as to be indistinguishable on the graph.) The experimental value is denoted by an asterisk.

charge radius predicted by this method is indicated by a full square ( $\mathbf{a})$. Experimental measurements are indicated at the physical pion mass by an asterisk $(\star)$. Note that for the charged baryons, two extrapolation schemes and two corresponding predicted physical values are shown, whereas (for the reasons explained in section III) only one extrapolation procedure is shown for each neutral baryon.

In the case of the proton, the two extrapolated results agree very well with the experimental measurement. It can be seen that a traditional linear extrapolation in $m_{\pi}^{2}$ would significantly underestimate the experimental result. Similarly the predicted charge radius for the neutron (produced by separate extrapolations of the $u$-and $d$-sector results) agrees with the experimental data significantly better than the prediction from a conventional linear extrapolation in $m_{\pi}^{2}$. Both predicted values for $\Sigma^{-}$also agree very well with the two experimental measurements. These baryons are currently the only baryons of the spin- $1 / 2$ octet whose electric charge radius has been measured.

From simple quark model arguments [9], where the heavier strange quark has a smaller distribution than the light quarks, we expect the hierarchy of electric charge radii of charged octet baryons to be given as follows

$$
\left|\left\langle r_{\Sigma^{+}}^{2}\right\rangle\right| \geq\left|\left\langle r_{p}^{2}\right\rangle\right| \geq\left|\left\langle r_{\Sigma^{-}}^{2}\right\rangle\right| \geq\left|\left\langle r_{\Xi^{-}}^{2}\right\rangle\right|
$$

Clearly the results of the lattice extrapolations shown in Table [ 


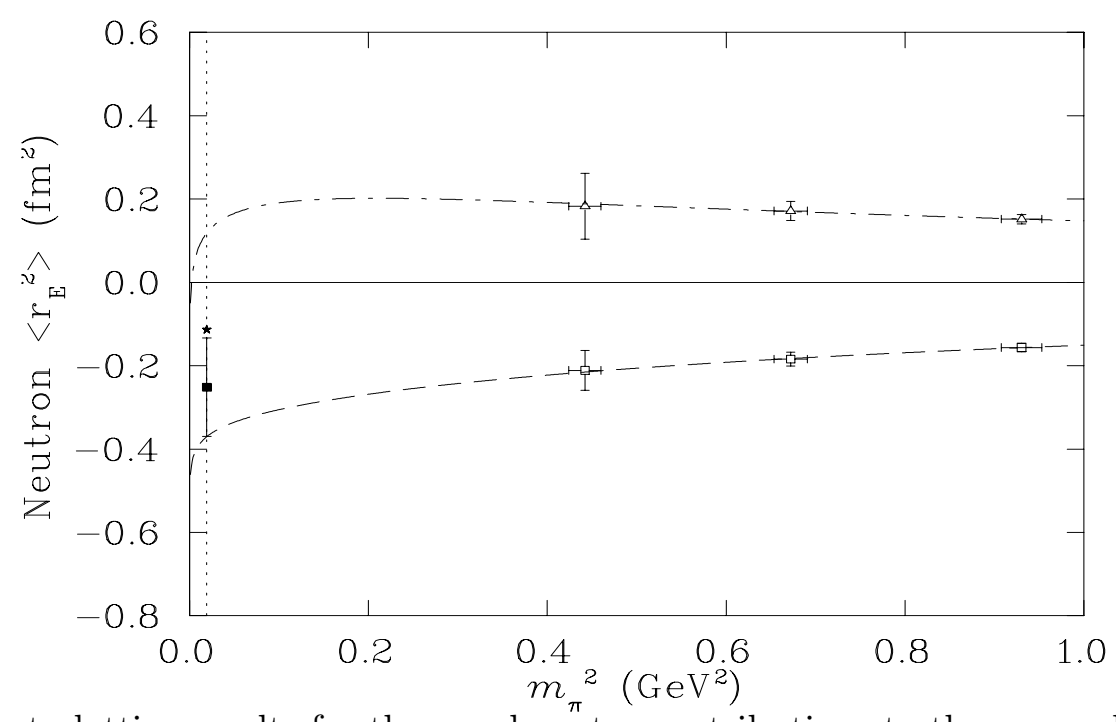

FIG. 3. Fits to lattice results for the quark sector contributions to the squared electric charge radius of the neutron. All symbols are defined in the caption below Fig. 2.

with this expectation. Indeed, in the regime of the actual lattice data $\left(m_{\pi} \geq 600 \mathrm{MeV}\right)$ the argument is even quantitative. However, as the chiral limit (and physical pion mass) is approached, the simple quark model description is no longer adequate and chiral physics gives rise to dramatic effects. For example, in the extrapolation of the $d$-quark sector of the proton (Fig. 2), chiral effects mean that the $d$-quark sector can actually make a positive contribution to the charge radius, via the $\bar{d}$ contribution in $\pi^{+}$- even though the total contribution is negative at the physical pion mass. This behaviour is not anticipated by the simple quark model.

For the neutral baryons the sign of the squared charge radius is important. In the neutron, the two $d$ quarks are most likely to be found in a spin 1 configuration, where they will undergo hyperfine repulsion. This leads to a small, negative charge radius squared. However, as one approaches the chiral limit, spontaneous chiral symmetry breaking, in particular the process $n \rightarrow p \pi^{-}$, which carries $d$-quarks to larger radii and screens the $u$-quark contribution via the $\bar{u}$ in the $\pi^{-}$, leads to an enhancement of the negative charge radius. The remaining neutral baryons, $\Sigma^{0}, \Lambda$ and $\Xi^{0}$, have a positive squared charge radius. This is because in each case the strange quark distribution is more localized than the light quark charge distribution (due to the larger mass of the strange quark). Therefore on average the light quark charge distribution occurs at a larger radius, resulting in a positive charge radius (since the light quark charge is positive in each case).

The lattice results used here were calculated with a strange quark mass of approximately $250 \mathrm{MeV}$ [9]. This is much larger than the physical strange quark mass of $115 \pm 8 \mathrm{MeV}$ at a scale $2 \mathrm{GeV}$, taken from a careful analysis of QCD sum rules for $\tau$ decay [13]. In an earlier study of lattice results for octet baryon magnetic moments [8] (where the results were extracted from the same lattice simulation [9]), it was found that the heavy strange 


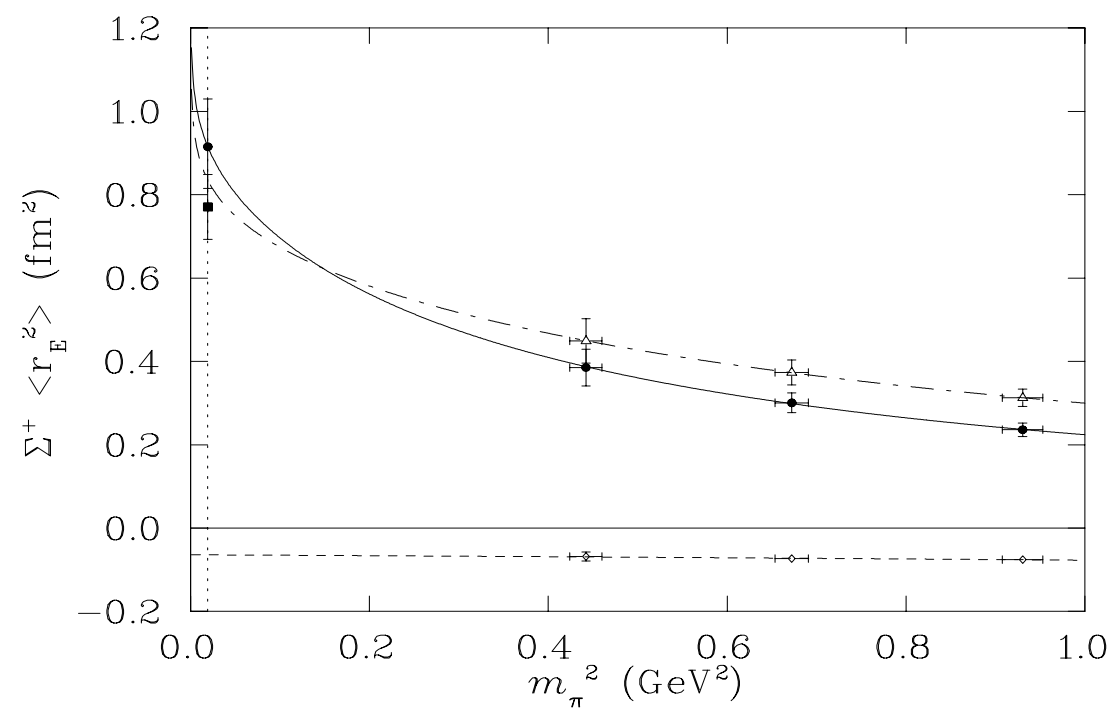

FIG. 4. Fits to lattice results of the squared electric charge radius of the $\Sigma^{+}$. Fits to the individual quark sector results are also shown. The strange quark sector results are indicated by open diamonds. All other symbols are defined in the caption below Fig. 2 .

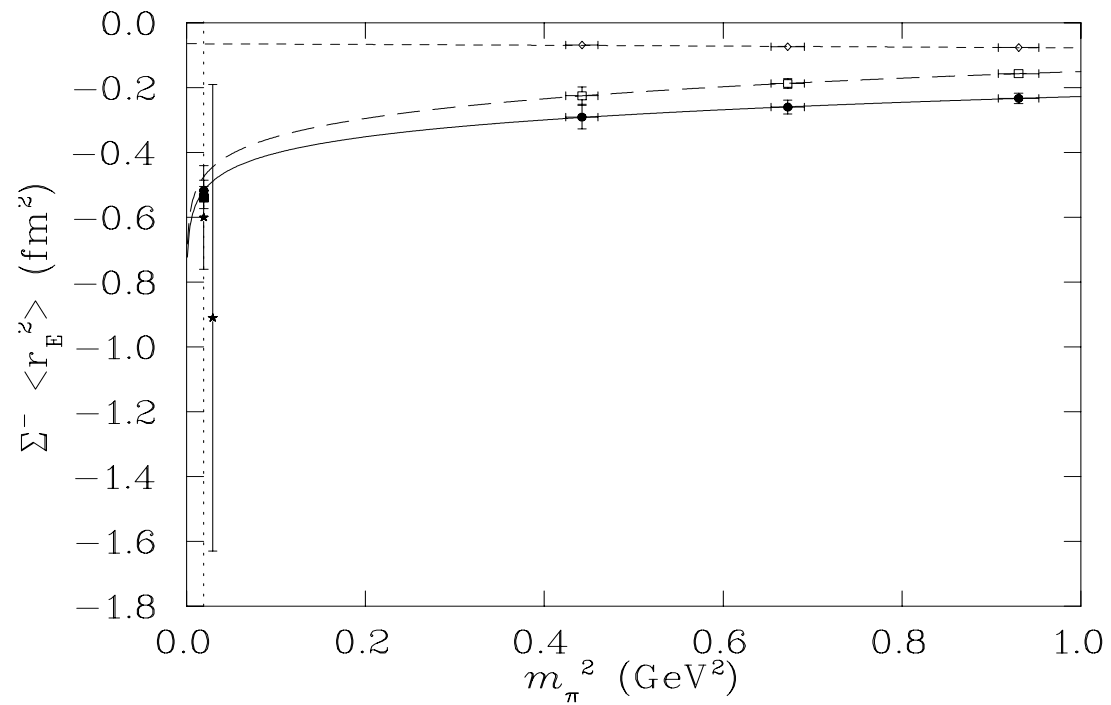

FIG. 5. Fits to lattice results of the squared electric charge radius of the $\Sigma^{-}$. Fits to the individual quark sector results are also shown. All symbols are defined in the captions below Figs. 2 and 1 . 


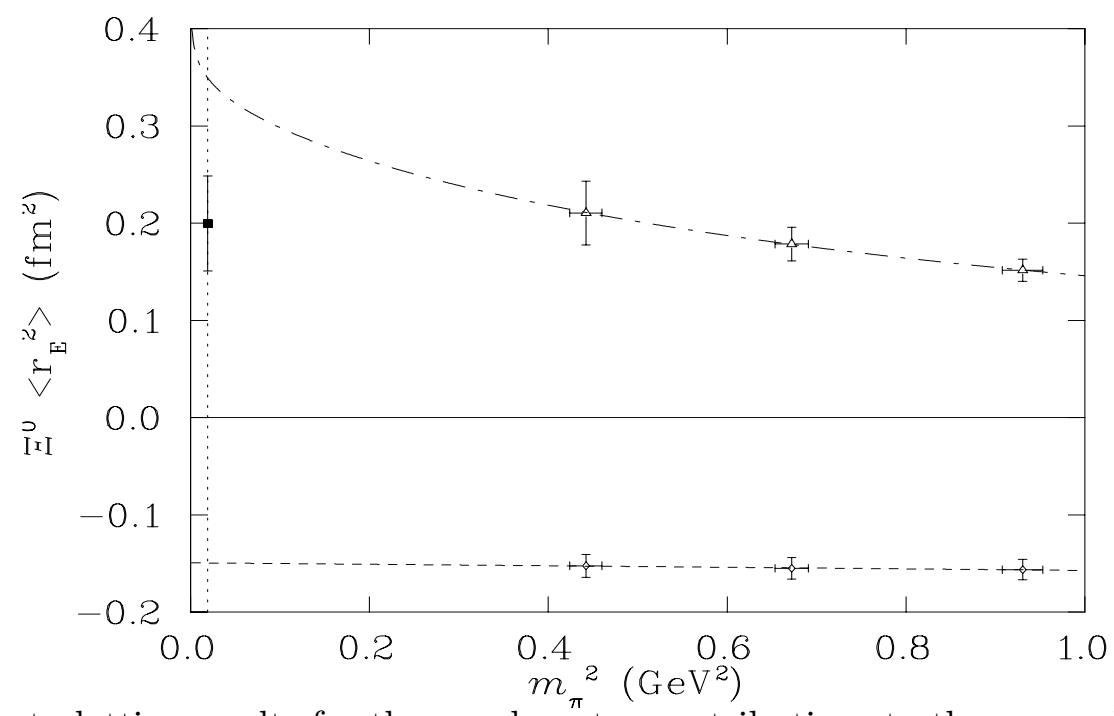

FIG. 6. Fits to lattice results for the quark sector contributions to the squared electric charge radius of the $\Xi^{0}$. All symbols are defined in the captions below Figs. 2 and 1 .

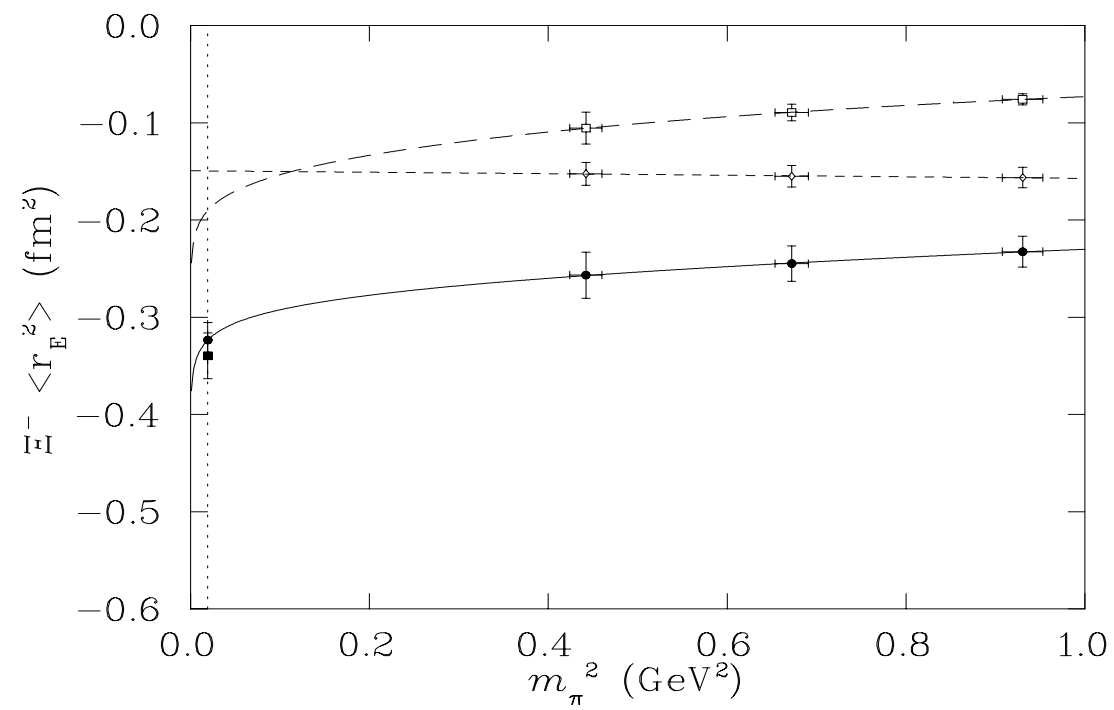

FIG. 7. Fits to lattice results of the squared electric charge radius of the $\Xi^{-}$. Fits to the individual quark sector results are also shown. All symbols are defined in the captions below Figs. 目 and 1 . 
quark had a significant effect on the predictions of the $\Xi$ moments. Here we expect that the heavy strange quark should also have some effect on the $\Xi$ charge radii. With a strange quark mass closer to the physical mass the strange quark contribution would be increased. This would result in a lower predicted charge radius for the $\Xi^{0}$ and a larger (in magnitude) charge radius for the $\Xi^{-}$. In the absence of experimental measurements we will not attempt to correct for the effect of the strange quark mass here.

As we see from Table [1, the extrapolated mean square charge radii obtained from both extrapolation procedures agree quite well for each charged octet baryon. For the proton, $\Sigma^{-}$ and $\Xi^{-}$the reconstructed values completely cover the result from the original extrapolations of Eq. (2). In the case of the $\Sigma^{+}$the two values overlap only on the error bars. This is due to the small variation in the strange quark contribution (which is caused by an environment effect). When this environment effect is included in the baryon charge radius, the magnitude of the slope is increased, resulting in a larger charge radius after extrapolation.

In turning the dimensionless masses calculated on the lattice to physical units, the lattice spacing, $a$, was set in the traditional manner by fixing the nucleon mass, obtained by a naive linear extrapolation in $m_{\pi}^{2}$, equal to the observed mass. Of course, such a linear extrapolation is known [5] to be inconsistent with chiral symmetry. We have checked that applying a more consistent chiral extrapolation would systematically lower values of $\left\langle r^{2}\right\rangle$ obtained for the charged octet by of the order 15\%. (The effect on neutral baryons is much smaller.) On the other hand, the data which we are forced to use is quenched data which omits some pion corrections. Although these are expected to be suppressed at the large values of $m_{\pi}^{2}$ for which the data is available (c.f. Ref. [⿴囗十), the associated systematic error would tend to increase the calculated values of $\left\langle r^{2}\right\rangle$, perhaps by $5-10 \%$. Rather than attempt to repair these deficiencies in the present data, we feel it would be more reasonable to simply accept that there is an additional systematic error of the order $15 \%$ associated with the extrapolated values shown in Table m. Adding this systematic in quadrature means that the values in Table 凹 would become, for example, $\left\langle r_{\star}^{2}\right\rangle=0.68 \pm 0.14 \mathrm{fm}^{2},\left\langle r_{\star \Sigma^{-}}^{2}\right\rangle=-0.54 \pm 0.09 \mathrm{fm}^{2}$, $\left\langle r_{\star \Sigma^{+}}^{2}\right\rangle=0.77 \pm 0.14 \mathrm{fm}^{2}$. We look forward to repeating our analysis with unquenched data at lower quark mass, which is the best way to overcome these problems.

\section{CONCLUSION}

In this paper we have investigated two methods of extrapolating lattice results for the electric charge radii of octet baryons to the physical regime. These procedures build in the correct leading non-analytic behaviour of the electric charge radii in the chiral limit, as well as the correct heavy quark behaviour. Both extrapolation procedures were performed for the charged octet baryons, with the predicted values agreeing very well. The extrapolation formulae seem to be very successful, as good agreement with experiment was obtained for the nucleons and the $\Sigma^{-}$. We await further experimental measurements of the baryon charge radii in order to test our predictions. In the future we hope to perform similar extrapolations of electric charge radius lattice results calculated with a more realistic strange quark mass and eventually with lighter, dynamical $u$ and $d$ quarks. 


\section{ACKNOWLEDGEMENT}

This work was supported by the Australian Research Council and the University of Adelaide. DBL would like to thank Frank Lee and the Center for Nuclear Studies at George Washington University for their kind hospitality during which some of this research was carried out. We would also like to thank Pierre Guichon for helpful comments on the manuscript. 


\section{REFERENCES}

[1] D.B. Leinweber and T.D. Cohen, Phys. Rev. D47, 2147 (1993) hep-lat/9211058.

[2] J.N. Labrenz and S.R. Sharpe, Phys. Rev. D54, 4595 (1996) hep-lat/9605034.

[3] M.F.L. Golterman and K.C. Leung, Phys. Rev. D58, 097503 (1998) hep-lat/9805032.

[4] D.B. Leinweber, D.H. Lu and A.W. Thomas, Phys. Rev. D60, 034014 (1999) heplat/9810005.

[5] D.B. Leinweber, A.W. Thomas, K. Tsushima and S.V. Wright, Phys. Rev. D61 074502 (2000) hep-lat/9906027.

[6] D.B. Leinweber and A.W. Thomas, hep-lat/9912052.

[7] D.B. Leinweber, A.W. Thomas and S.V. Wright, hep-lat/0001007.

[8] E.J. Hackett-Jones, D.B. Leinweber and A.W. Thomas, hep-lat/0004006.

[9] D.B. Leinweber, R.M. Woloshyn and T. Draper, Phys. Rev. D43, 1659 (1991).

[10] B. Kubis, T.R. Hemmert and U. Meissner, Phys. Lett. B456, 240 (1999) hepph/9903285.

[11] M. Bae and J. A. McGovern, J. Phys. G G22 (1996) 199 hep-ph/9509366.

[12] K. A. Aniol et al. [HAPPEX Collaboration], Phys. Rev. Lett. 82 (1999) 1096 nuclex/9810012.

[13] K. Maltman, Phys. Lett. B462, 195 (1999) hep-ph/9904370.

[14] P. Mergell, U. Meissner and D. Drechsel, Nucl. Phys. A596, 367 (1996) hep-ph/9506375.

[15] S. Kopecky et al., Phys. Rev. Lett. 74, 2427 (1995).

[16] I. Eschrich (for the SELEX collaboration), hep-ex/9811003.

[17] M. I. Adamovich et al. (for the WA89 Collaboration), Eur. Phys. J. C8, 59 (1999).

[18] N. Cabibbo and E. Marinari, Phys. Rev. Lett. 119B, 387 (1982).

[19] B. Efron, SIAM Rev. 21, 460 (1979).

[20] S. Gottlieb, P. B. MacKenzie, H. B. Thacker, and D. Weingarten, Nucl. Phys. B263, 704 (1986). 